\title{
Tungsten Lamps of High Efficiency-I ${ }^{*}$
}

\author{
The Loss of Efficiency of Tungsten Lamps By Blackening of the Bulb, and Methods of Preventing It
}

\author{
By Irving Langmuir
}

WhiLe the transformation of electrical energy into heat or even into mechanical energy has for many years heen accomplished with efficiencies well above 90 per cent, the artificial production of light has been notoriously inefficient.

The first carbon incandescent lamps had efficiencies of five to six watts per mean horizontal candle, but were gradually improved over a long period of years to about 3.1 watts per candle, until finally by the use of the 3.1 watts per candle, until finally by the use of the
metalized filament an efficiency of 2.5 watts per candle was reached commercially. Since the introduction of the metalized carbon filament, progress with othe types of lamps has been comparatively rapid, and at the present time the most efficient commercial incandescent lamn, the lamp with a tungsten filament, has an ef ficiency from one to one and a quarter watts per candle in the ordinary sizes.

Notwithstanding this decided improvement, we ar still far from the theoretical maximum of efficiency that would be attained if all the energy of an electric circui were converted into visible light. Drysdale has shown that perfect efficiency for the production of white light would be atout 0.10 watt per candle, whereas with the production of a monochromatic yellow-green light, th efficiency would reach as high as 0.06 watt per candle.

The luminous efficiency of the ordinary tungsten lam is therefore not better than about 6 to 10 per cent.

The causes which have made it necessary to operat tungsten lamns at such relatively low efficiencies as on watt per candle have been little understood. It seemed, therefore, that an investigation of the phenomena occurring in tungsten lamps, carried out with a view of reaching a clear understanding of the causes of the failure of the lamps, might possibly open the way to the discovery of methods by which the efficiency could be greatly improved.

The present paper is a description of some experimental and theoretical work, extending over a perio of many years, which has now resulted in the produc tion of a new type of tungsten lamp; a lamp which will give a life of more than 1000 hours, at an efficiency in the neighborhood of 0.50 watt per candle.

INVESTIGATION OF CAUSE OF FAILURE OF ORDINARY TUNGSTEN LAMPS.

Ordinary tungsten lamns fail, in general, in one of two wavs; namely, either hy breakage of the filaments, or hy blackening of the bulb. In ordinary practise the useful life of the lamp is considered to be the tim which the lamp burns before its candle-power has fallen to 80 per cent of its original value, or until the filament breaks, in case this occurs while the candle-power is still above 80 per cent.

The breakage of the filament was a very serious factor in the early tungsten lamps, as the filament materia was extremely brittle. This difficulty has now been overcome by the nroduction of ductile tungsten wire and by hetter methods of mounting the wire in the bulbs, so that lamps ean now be made which are so strong that hlow is more likely to break the bulb than the filament.

The life of tungsten lamps is therefore at present practically determined by the rate at which the candlenower decreases. The main cause of this decrease i nower decreases. The main cause of this decrease is several hundred hours. It is due simply to the blackening of the inner surface of the bulb.

The cause of this blackening was the subject of much peculation. The prevalent oninion seemed to be that in a normallv operated lamp it was due to disintegration of the filament, caused by the presence of traces of residual gas, whereas in lamps run at much more than their rated voltage it was perhaps caused by evanoration. Others, however, considered it due to leakage currents of electricity (Fdison effect) across the space currents of electricity (Fdison effect) across the space
hetween the positive and negative end of the filament hetween the positive and negative end of the filament Still others were of the opinion that the blackening of the bulb wasd ue primarily to evaporation of the filament.

In the manufacture of carbon incandescent lamps, it had been found necessary to use a relatively high vacuum, as otherwise the lamns were found to have a very short life. That is, the bulbs blackened rapidly or discharge occurred between the two ends of the filament, finally occurred between the two ends of the filament, finally
resulting in the formation of an arc which destroyed resulting in the formation of an arc which destroye
the lamp. Various attempts had been made to prevent the blackening by the introduction of gases at variou pressures in the lamp. For example, Edison propose introducing nitrogen or cyanogen at relatively hig pressures into lamp bulbs, for the purpose of preventing

* A papar presented at the 286th meeting of the American Institute of Electrical Engineers. New York, October 10th, 1913. the electric discharge between the positive and the negative end of the filament. In this way he hoped to prevent blackening. These attempts, however, were completely unsuccessful, and it can be readily shown in
the case of a carbon lamp, run at say 3 watts per candle and containing nitrogen at atmospheric pressure, that the filament loses weight more rapidly than when run in a vacuum at the same efficiency.

In the commercial production of lamps it was found necessary at first to use mercury pumps for the exhaustion of the lamps; mechanical pumps were not good enough. Later it was found possible to obtain a sufficiently good vacuum with mechanical pumps, which by that time had been considerably improved, by introducing red phosphorus into the lamp just before sealing off, and at the same time heating the filament to a much higher temperature than that at which it was to run normally. It should be pointed out that not only was it necessary to use some such special method of exhaust, but in order to obtain lamps of good life, the bulbs themselves had to be heated to a high temperature during the exhaust, in order to drive off any gases condensed on the surface of the glass. It is interesting to notice that the lamp manufacturers had adopted these precautions in obtaining a high vacuum long before the necessity for them was realized by most scientific investigators engaged in work with high vacuum.

When the lamp factories began the manufacture of tungsten lamps, they found that much greater precautions were needed in the exhaustion of these lamps than had been necessary for the ordinary carbon lamps, and many improvements in the methods of exhaustion were adopted.

Unless all this care was taken to obtain the best possible vacuum, there were striking evidences of the presence of

Various attempts to improve the life of lamps hy obtaining a better vacuum than usual had not been very successful. This failure, however, could not be taken as proof that a better vacuum would not improve the life In the first place, it had been found that the vacuum of lamp gradually improved after sealing off ("clean-up" effect), the pressure finally reaching a value probably lower than that directly obtainable by any of the well known methods of exhausting. Yet even where we had pressures lower than would be indicated by the most sensitive vacuum gages, we often had clear indication that the blackening of the bulb was due to imperfect exhaust. It seemed quite possible, therefore, that there might remain, in lamps, minute traces of some gas or vapor which we had not yet learned to remove by our usual methods of exhaust. This residual gas might easily be the cause of the gradual blackening of the bulbs.

Since the pressures were too low to measure, we had no way of definitely knowing whether one method of exhaust was better than another, so that any failure to improve the life by a new method of exhaust might simply mean that the vacuum had not been improved.

It seemed, therefore, that the question as to whethe a better vacuum would give a better lamp could only be settled by a direct investigation of the cause of the blackening.

Two lines of attack were decided upon:

1. Study of the sources of gas within a lamp.

2. Effects produced in lamps by various gases. SOURCE OF GAS WITHIN THE LAMP.

There are four sources of gas within the lamp bulb: first, residual gas left by evacuation; second, gas given off by the filament; third, gas from the lead-in wire or the anchors; and fourth, the gas given off by the glass. 1. Residual Gas. The mechanical pumps ordinarily used in exhausting lamps produce a vacuum of about 0.001 millimeter, according to the McLeod gage. Thi is probably about the pressure of the non-condensible gases left in the lamp. Besides this, however, there must be some water vapor and oil vapor, and if the filament has been lighted during the exhaust, as is usually the case, there will be some carbon monoxide, carbon dioxide, and hydrogen produced by the action of the filament on the vapors. Probably most of these gases are nearly completely removed, or precipitated on the walls of the bulb, by the clean-up that occurs when the phosphorus is volatilized into the lamp and a blue glow made to occur. The final pressure, just after sealing off, is usually in the neighborhood of 0.001 millimeter or less.

2. Gas from the Filament. The prevalent opinion, as expressed generally in scientific literature, is that metals when heated to very high temperature in vacuum, evolve very large quantities of gas. For example, in a
recent article, Prof. J. J. Thomson (Nature, 91, p. 335, 1913) says: "Belloc, who has recently published som interesting experiments on this subject, after spending about six months in a fruitless attempt to get a piece of iron in a state in which it would no longer give off gas when heated, came to the conclusion that, for practical purposes, a piece of iron must be regarded as an inexhaustible reservoir of gas." Thomson's own experience is quite similar.

The first few experiments on the gases evolved from the filament of a tungsten lamp also seemed to show the presence of inexhaustible supplies of gas within the filament. Later work proved, however, that this gas was not actually evolved from the filament, hut was produced from the decomposition by the filament of water vapor or hydrocarbon vapors present at extremely low pressure in the bulbs. It was finally found that with small filaments, such as are used in lamps, the gas evolved by heating is not more than from three to ten times the volume of the filament. By thoroughly cleaning the surface of the wire hefore heating, the amount of gas is usually not over half as great. The surprising fact was observed that at least 90 per cent of the gas was given off within a few seconds on first heating the wire to a temperature exceeding $1500 \mathrm{deg}$. Cent. At a temperature below 1,200 deg. however, the gas is given off only very slowly, if at all. The gas consists of about 70 to 80 per cent carbon monoxide, the remainder being mostly hydrogen and carbon dioxide. The total amount of gas evolved from the filament of a 40-watt lamp, if liberated in the lamp after sealing off, would produce a pressure of from 0.006 to 0.02 millimeter.

3. Gas from Lead-in Wires and Anchors. In many of the larger lamps, where the leads or anchors become very hot, there are often clear indications that the gas evolved from this source has a marked effect on the life, particularly on the tendency to arc across during aging. In the experimental lamps made with small sizes of wire, the quantities of gas obtained from this source were found to be too small to measure.

4. Gas from the Bulb. On heating bulbs of 40-watt lamps for three hours to a temperature of $200 \mathrm{deg}$. Cent., after having dried out the bulhs at room temperature for 24 hours hy exposure in a good vacuum to a tube immersed in liquid air, the following quantities of gas were given off:

200 cubic millimeters water vapor.

5 cubic millimeters carbon

These are the quantities of gas, liberated by the heating, expressed in cubic millimeters at room temperature and atmospheric pressure.

By raising the temperature of the bulbs from 200 deg. to $350 \mathrm{deg}$., an additional quantity of water vapor was obtained, so that the total now became.

300 cubic millimeters water vapor.

20 cubic millimeters carbon dioxide.

4.cubic millimeters nitrogen.

A subsequent heating of the bulbs to $500 \mathrm{deg}$. Cent. caused the total amount of gas evolved to increase up to 450 cubic millimeters water vapor. 30 cubic millimeters carbon dioxide. 5 cubic millimeters nitrogen.

At each temperature the gas stopped coming off the glass after a half hour of heating, only to begin again whenever the temperature was raised to a higher value than that to which the bulb had been previously heated. It therefore seems that even by heating the bulb to 500 deg. not all of the water vapor can be removed, but it does seem probable that after this treatment the amount of water vapor that can come off a bulb at ordinary temperatures must be extremely small.

This study of the origin of the gases within a lamp thus led to the following important conclusion: The amounts of residual gas, together with all the gas that is given off by the filament and its supports, are quite insignificant as compared with the gas on the inner surface of the bulb. Furthermore, the great difficulty of completely removing the gases from the glass makes this source particularly troublesome in incandescent lamps. We see that the gases likely to be present or given off in an exhausted lamp are, in the probable order of their importance: water vapor, carbon dioxide, hydrocarbon vapors, hydrogen, carbon monoxide, nitrogen and, when phosphorus is used, various phosphorus compounds. 
EFFECTS PRODUCED IN LAMPS BY VARIOUS GASES.

Hydrogen. This gas cleans up (disappears) in a lamp bulb in four distinct ways. Relatively large quantitie (20 to 50 cubic millimeters) of hydrogen are driven on to the bulb when the filament is at relatively low temperature (1,500 deg. or more). This hydrogen is particularly active chemically (atomic hydrogen) and will react even at room temperature with many reducible substances. Moderate heating of the bulb will cause a large part of it to escape from the glass again. Since water vapor in the bulb is decomposed by the filament to form hydrogen and an oxide of tungsten, there is nearly always a considerable amount of active hydrogen stored up on the bulb after the lamp has been running some time.

The amount of heat carried away from a filament by hydrogen at low pressures, say 0.001 millimeter, although many times greater than with any other gas, was found to be entirely negligible compared with the heat radiated from the filament. The cooling effect of such pressures from the filament. The cooling effect of such pressures
of gas, therefore, has no apprecialle effect on the life of lamps, even though, as is usually the case, the lamps are set $u p$ at a given efficiency.

Dry hydrogen in lamps was never found to have the slightest tendeney to produce. blackening of the bullss. That is, the bulbs never blackened more rapidly than if the filament were run at the same temperature in a vacuum. Subsequent experiments have proved that this is true from low pressures up to atmospheric pressure.

-xygen. At all temperatures above 1,000 deg. this
gas reacts with tungsten to form the yollow oxide $\mathrm{WO}_{3}$, no matter how low the pressure of the oxygen may be. The oxide distills off the filament and deposits on the bulb, but owing to its light color the deposit is invisible when the amount of oxygen is less than 100 to 200 cubic millimeters. Oxy

Nitrogen. There are three ways in which this gas cleans up in a lamp, each being an exceptionally interestcleans up in a lamp, each being an exceptionally interesting phenomenon in itself. With voltages above 40
volts and pressures above 0.001 millimeter the nitrogen cleans up provided the filament temperature exceeds 2,000 deg. and causes an attack of the negativo and of the filament, producing a brown deposit of tungsten nitride, $\mathrm{WN}_{2}$, on the bulb. Except where the amount of nitrogen that cleans up is much larger than could possibly be present in an ordinary lamp this gas never causes any discoloration of the bulb.

Carbon Monoxide. This gas behaves almost exactly like nitrogen. At low pressures it never produces perceptible blackening of the bulb, although at higher pressures it may slowly give a slight deposit of earbon under certain conditions. The results, however, clearly
indicated that traces of carbon monoxide such as might indicated that traces of carbon monoxide such as might exist in lamps could not be responsible for the blackening.

Carbon Dioxide. This gas attacks the filament and produces carbon monoxide and an oxide of tungsten, without producing any perceptible blackening.

Water Vapor. Even very low pressures of water Water Vapor. Even very low pressures of wate
vapor react with the tungsten filament in a lamp to produce hydrogen, and cause rapid hlackening of the bulb. Thus a lamp made up with a side tube containing a little water which is kept cooled by a freezing mixture of solid carbon dioxide and acetone (-78 deg. Cent.) will blacken very rapidly when running at normal temperature is only about 0.0004 millimeter.

The fact that lamps exhausted at low temperature (say 100 to 200 deg.) blacken so rapidly during life, together with the fact that water vapor is the principal gas removed from the bulb by heating, indicate that the
water vapor is responsible for the short life under these conditions.

It is rather surprising that water vapor should have hydrogen or oxygen, acting alone, produces no blackening.

The explanation of the behavior of the water vapor seems to be as follows:

The water vapor eoming into contact with the filament is decomposed, the oxygen combining with the tungsten and the hydrogen being coolved. 'The oxide distills to the bulb, where it is subsequently reduced to metallic tungsten by atomic hydrogen given off by the filament, water vapor being simult aneously produced. limited quantity of water vapor.

Several experiments indicated that the amount of Several experiments indicated that the amount of
tungsten that was carried from the filament to the bulb was often many times greater than the chemical equivalent of the hydrogen produced, so the deposit on the
bulb could not well be formed by the simple attack of bulb could not well be formed
the filament by water vapor.

Another experiment demonstrated that even the yellow oxide, $\mathrm{WO}_{3}$, could be reduced at room temperature by atomic hydron. A flament was heated in a well exhausted bulb containing a low pressure of oxygen;
this gave an invisible deposit of the yellow oxide on the this gave an invisible deposit of the yellow oxide on the
bulb. The remaining oxygen was pumped out and dry hydrogen was admitted. The filament was now lighted to a temperature $(2,000 \mathrm{deg}$. K.) so low that it
could not possibly produce blackening under ordinary could not possibly produce blackening under ordinary
conditions. In a short time the bulb became distinctly dark, thus indicating a reduction of the oxide by the active hydrogen. Further treatment in hydrogen failed to produce any further darkening, showing that the oxide could only be reduced superficially. Mercury Vapor. Contrary to earlier experiments,
it was found that mercury vapor in a lamp did not cause it was found that mercury vapor in a lamp did not cause
blackening if the voltage was low enough so that no blackening if the voltage was
serious Edison effect occurred.

\section{ATTEMPTS TO ELIMINATE WATER VAPOR.}

This study of the effects produced by various gase led to the conclusion that if the blackening of bulbs of ordinary lamps was caused by imperfect vacuum, then it must be due to water vapor and the further life of the lamps. The problem of improving the eflife of the lamps. The problem of improving th
ficiency of lamps thus assumed more definite form.

ficiency of lamps thus assumed more definite form.
Lamps were exhausted in a special vacuum oven, that the temperature of the bulb could be raised during exhaust to a temperature aloout $100 \mathrm{deg}$. higher than that otherwise attainable. A good mercury pump was used and care was taken to remove the last traces of mercury vapor, water vapor and carbon dioxide, by placing between the lamp and the pump a trap immersed in liquid air. 'The lamps were exhausted from one to three hours under these conditions. The filaments were hours under these conditions. The filaments were
heated to high incandescence to drive off gas. The heated to high incandescence to drive off gas. The
lamps were sealed off when the pressure by the McLeod gage read about 0.00005 millimeter. These lamps were put on life test and compared with other lamps made under factory conditions.

The unexpected result of this work was that the life of the lamps exhausted with all these precautions was not materially better than the best of the lamps made regularly in the factory. The life of the lamps could certainly not be improved on the average by more than 20 per cent by such methods.

Very special methods of exhaust did not improve the life of the lamp above that of an ordinary lamp run under normal conditions, but they did make it possible for a lamp to run with the bulb at a high temperature without serious impairment of its life. This seemed to demonstrate that even the complete removal of water vapor from the lamp bulb would not lead to a very radical improvement in the life of the lamp, although the presence of minute traces of water vapor certainly the presence of minute traces of water

The conclusion to be drawn from all of the foregoing work is that the blackening of the bulbs of ordinary well made and well exhausted lamps is not caused by imperfect vacuum.

Among all the causes of the blackening that have tion of the filament.

EVAPORATION OF TUNGSTEN.

To test out whether or not this was the correct explanation, many experiments were undertaken to dewhen run at various temperatures in lamps. It was found that in lamps with filaments run at the same temperature the loss in weight was proportional to the surface of the filament and independent of the size of the bulb. The temperature coefficient of the rate of if it were proportional to vapor pressure of the metal.

Furthermore, the actual measurements at various Furthermore, the actual measurements at various
temperatures agreed remarkably well with the rational formula for vapor pressure:

$$
\log P=A-\frac{B}{T} C \log T
$$

Experiments with lamps exhausted at a low temperature have shown that the temperature coefficient of the rate of blackening is much lower than in well exand run at say 5 watts per candle, often blacken nearly and run at say 5 watts per candle, often blacken nearly
as quickly as similar lamps run at 2 watts per candle, as quickly as similar lamps run at 2 watts per candle, be very different. This serves to show clearly the radical difference between the two kinds of blackening.

METHODS OF PREVENTING THE BLACKENING OF BULBS. Having now shown that the blackening of ordinary tungsten lamps is caused by evaporation of the filament, becomes a very definite one.

Introduction of Gases at High Pressure. Although in Introduction of Gases at High Pressure. Although in
the past it has usually been found that the presence of a high pressure of gas causes an increase in the rate of disintegration of a heated metal, yet if we know, as we now do in the case of tungsten, that the phenomenon is simply one of evaporation, then we have every reason to believe that the presence of a chemically inert gas will reduce this evaporation. We have seen that low produce any perceptible blackening of the bulb, and produce any perceptible blackening of the bulb, and
therefore produce no disintegration in the ordinary sense.
Most gases react chemically with tungsten at high
temperature, but hydrogen, nitrogen, argon, and mercury temperature, but hydrogen, nitrogen, argon, and
vapor seem to be chemically inert toward it.

In the manufacture of tungsten filaments it was for a ong time the practice to sinter the filament thoroughly by heating it to a high temperature in hydrogen or in a mixture of nitrogen and hydrogen. If care were taken to avoid air or moisture in the "forming gas" the filaments would stand heating for a long time in these gases, which indicated that they were at least relatively chemically inert.

Whether the loss in weight at a given temperature was actually greater or less than in vacuum could not be determined from these rough observations. To test this out, a lamp was made and filled with carefully dried and purified hydrogen at atmospheric pressure. The filament was run at the same temperature as that of lamps running at one watt per candle. The heat loss from the filament by ronvection was so serious that loss from the filament hy ronvection was so serious that actually 17 watts per eandle were required to maintain
the filament at this temperature. This lamp, however, ran for more than 36i) hours without showing any blackening of the bull, or any greater loss of material from the filament than would have been the ease in vacuum at the same temperature. This result was very striking, as the bull was rumning so hot that the life of a filament in vacuum, in a bulb at the same temperature, would have been very short indeed. Subsequent experiments fully confirmed the first one, and showed that even in the presenes of hydrogen at atmospheric pressure, the loss of weight of tungsten was much less than in vacuum. The loss of heat, however, was so great that it would he entirely impracticable to make a lamp with the tungsten filament in hydrogen at high pressure. Subsequent experiments showed, however, that the heat conductivity of hydrogen at very high temperature was abnormally great --much greater than would be expected from the ratio of its heat conductivity to that of other gases at room temperature. 'This is due to the
fact that at high temperatures hydrogen becomes disfact that at high te
sociated into atoms.

Experiments were then tried in nitrogen at atmospheric pressure. Nitrogen was found to be cntirely inert towards the tungsten, and to conduct so little heat that with a fairly large diameter filament the ceffieiency was as high as 0.24 watts per candle, at a temperature close to the melting point of tungsten. 'The rate of evaporation was found to be much less than in vacuum.

The next point to be determined was whether the The next point to be determined was whether the
decrease in evaporation was sufficient to off set the heat lost by convection. Berause of the presence of the gas, the temperature of the filament was run considerably higher than in vacuum, in order to obtain the sam efficiency. Whether or not the rate of evaporation in gas at this higher tcmpcralure would be less than the rate of evaporation in vacuum at the same efficiency, is a point to be determined only be experiment. A careful study was therefore undertaken of the
laws of heat convection from filaments at high temperalaws of heat convection from filaments at high tempera-
ture in various gases, since the knowledge on this subject was extremely meager. Experiments were made with platinum wires in air, with platinum wires in carbon dioxide and in hydrogen, and with tungsten wires in hydrogen, nitrogen and mercury vapor.

According to the formulæ developed in the course of this work, the heat loss from wires of any size in any of the ordinary gases at any temperature could be calculated. In this way it was found that the loss of efficiency (at constant temperature) due to the introduction of a gas at high pressure is very much greater for filaments of small size, than for the larger ones, so that with wires of the sizes ordinarily used in lamps the temperature would have to be raised excessively in order to obtain an efficiency of even one watt per candle. The advantages of a large diameter filament can be practically obtained by coiling a smaller wire into a tightly wound helix.

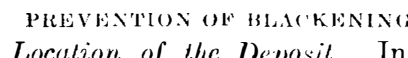

Changing Loculion of the beposil. In lamps with a very high vacuum, the atoms of tungsten as they are given off from the filame'nt ly evaporation, travel in straight lines until they strike the bulb. As they are electrically uncharged (this has been demonstrated by experiment), the ficld produced by the filaments has no influence on the location of the deposit. Since the light from the filament also travels in straight lines, according to similar laws, it follows that in a high vacuum the deposit always collects most on those portions of the bulb where the strongest light passes through the glass. In an imperfect vacuum, especially in the presence of argon, the tungsten atoms tend to become negativel charged and depesit on the bulb very irregularly.

With pressures of nitrogen less than 50 millimeters,
the brown deposit of nitride is distributed over the bulb in much the same way as the tungsten deposit in ordinar lamps. $\Lambda t$ higher pressures than this the effects of convection currents become apparent. At atmospheric pressure this effect is very striking. ('To be continued.) 